\title{
Budgerigar Fibrosarcoma
}

National Cancer Institute

\section{Source}

National Cancer Institute. Budgerigar Fibrosarcoma. NCI Thesaurus. Code C134943.

Fibrosarcoma that occurs in a budgerig ar. 